\title{
评述
}

\section{具有活性氧清除能力的功能纳米材料用于急性肾 损伤缓解}

\author{
侯林倩, 张瑞, 仲晓燕, 程亮 ${ }^{*}$ \\ 苏州大学功能纳米与软物质研究院, 苏州纳米科技协同创新中心, 苏州 215123 \\ * 联系人, E-mail: lcheng2@suda.edu.cn \\ 收稿日期: 2020-07-26; 接受日期: 2020-10-19; 网络版发表日期: 2021-04-15 \\ 国家自然科学基金(批准号: U20A20254, 52072253)资助
}

摘要 急性肾损伤(acute kidney injury, AKI) 是一种高发病率、高死亡率的危重疾病. 除了支持性治疗, 如血液透 析和肾脏移植之外, 暂时无针对性的治疗方法. 因此需要从AKI发病机制着手, 探索一种新型的治疗策略. 近期研 究表明, 氧化应激是急性肾损伤的重要病理机制之一。因此, 有针对性地缓解肾脏氧化应激成为AKI治疗的关键. 具有还原性的功能纳米材料的发展为治疗急性肾损伤提供了新的思路和可能的途径。本文综述了近年来具有活 性氧清除能力的功能纳米材料在AKI治疗中的研究进展.

关键词急性肾损伤, 还原性材料, 活性氧清除, 纳米颗粒

急性肾损伤(acute kidney injury, AKI)也称为急性 肾功能衰竭, 是一种常见的临床疾病 ${ }^{[1 \sim 3]}$, 临床表现为 肾排泄功能迅速下降，氮代谢积累以及尿量的减少. AKI的发病率和死亡率极高，全世界每年有 1330 万 $\mathrm{AKI}$ 新病例、 200 万死亡病例 ${ }^{[4]}$. AKI可由多种损伤引 起，包括缺血再灌注、败血症、低血压以及抗生素和 化疗药物过量使用等, 可能会致使肾脏产生过量活性 氧(reactive oxygen species, ROS). 在正常情况下肾脏 可以耐受代谢产生的少量ROS. 然而过量ROS使得细 胞发生氧化应激损伤，细胞调亡，从而进一步刺激间 质成纤维细胞的活化，导致肾纤维化，进而形成慢性 肾脏疾病, 最终完全丧失肾脏功能 ${ }^{[5]}$. 除了支持性治 疗, 如血液透析和肾脏移植外 ${ }^{[6,7]}$, 目前尚无有效的临
床治疗方法. 因此, 清除肾脏部位产生的过量ROS可作 为 $\mathrm{AKI}$ 治疗的关键 ${ }^{[8]}$.

材料学的发展为AKI的治疗提供了新的思路, 大 多数药物小分子在体内的半衰期较短, 应用纳米载体 可以实现在体内长时间循环, 提高药物的生物利用度 和疗效 ${ }^{[9,10]}$. 纳米颗粒还可以控制药物释放, 在颗粒到 达目标组织后释放大量药物, 达到药物靶向释放的效 果. 此外, 具有还原性的纳米材料能够很好地清除 ROS, 也可以用于AKI的治疗. 这些纳米材料在平衡细 胞内氧化应激水平的同时也能通过肾脏代谢排出，从 而降低其对全身系统的毒性. 本文主要综述具有ROS 清除能力的功能纳米材料在AKI疾病治疗中的应用 (图1). 


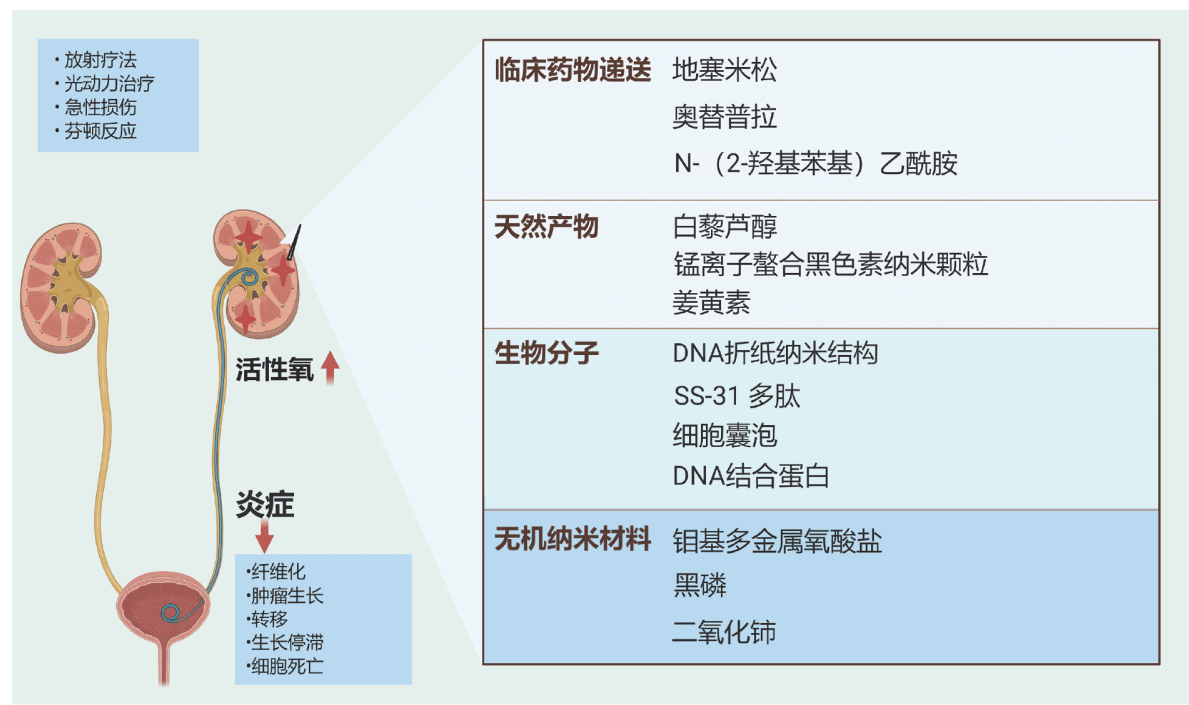

图 1 四类材料在AKI模型中的应用(网络版彩图)

Figure 1 Applications of four kinds of materials in AKI model (color online)

\section{1 具有活性氧清除能力的功能纳米材料用 于急性肾损伤缓解}

\section{1 临床药物递送}

在AKI治疗过程中，已经有多种药物进入临床使 用 ${ }^{[1]}$, 而药物使用过程中没有特异性，需要频繁给药， 从而不可避免地出现全身毒性. 纳米材料的出现一定 程度上解决了这一问题. 通过构建纳米药物递送系统 实现药物的高效和有针对性的递送，降低了全身性的 毒性, 从而改善了治疗效果. 在AKI疾病模型中, 中性 粒细胞附着在活化的内皮细胞上产生活性氧，从而导 致管状上皮和内皮细胞完整性降低，进而导致肾脏功 能受损．受损的管状上皮细胞会释放促炎因子和趋化 因子，如肿瘤坏死因子- $\alpha$ 、白细胞介素- 6 、白细胞介 素- 8 等 ${ }^{[11,12]}$, 这些因子有助于招募免疫细胞. 同时上皮 细胞能够表达黏附分子、Toll样受体(Toll-like receptors, TLRs)和T细胞共刺激分子, 它们激活免疫细胞并 放大炎症反应. 因此, 在临床治疗中经常使用抗炎药物 缓解AKI患者的肾脏炎症.

地塞米松(dexamethasone, DXM)有抗炎、抗氧化 的作用, 已在临床上广泛使用 ${ }^{[13,14]}$. 2017年, $\mathrm{Hu}$ 等人 ${ }^{[15]}$ 制备了负载地塞米松的唾液酸修饰的固体脂质纳米颗 粒(sialic acid-conjugated polyethylene glycol (PEG) ylated nanoparticle, SA-NP), 其在生理环境下有良好 的稳定性(图2A). SA-NP可通过下调氧化应激诱导的
Bax，上调Bcl-xL，抑制Caspase-3和Caspase-9的活化来 降低细胞凋亡．炎症血管内皮细胞表达的e-选择素受 体可与唾液酸特异性结合, 因此, 利用炎症血管内皮 细胞能够特异性内化SA-NP, 从而实现药物的靶向递 送. 采用近红外染料ICG(indocyanine green)标记SANP研究肾脏靶向性. 结果表明, SA-NP处理后肾脏组 织有非常高的地塞米松的积累, 同时SA-NP在不同器 官的分布表明, SA-NP减少了药物的非特异性分布, 从而能够有效改善AKI小鼠血液生化指标、组织病理 学、氧化应激水平, 实现了药物在特定组织释放, 延长 了药物作用时间, 有效地改善了治疗效果, 减少了药物 的不良副反应.

肾脏在发生氧化应激损伤后会产生严重纤维化。 2019年, Yu等人 ${ }^{[16]}$ 将治疗肾纤维化药物奥替普拉(oltipraz)装载在聚乳酸-乙醇酸(poly lactic-co-glycolic acid, PLGA)纳米载体中(图2B), 构建了直径 $100 \mathrm{~nm}$ 左右的 纳米颗粒(PLGA-Oltipraz NPs). PLGA-Oltipraz NPs可 选择性地在AKI小鼠肾脏中累积. 随后药物逐渐释放, 激活抗氧化应激相关的 Nrf2 及其下游靶点 NQO1, GCLC, Gpx2的表达，从而减轻肾小管损伤和肾纤维 化. 与游离的奥替普拉处理组相比, PLGA-Oltipraz NPs处理组有较低的丙二醛(malondialdehyde, MDA) 和更高的超氧化物歧化酶(superoxide dismutase, SOD) 水平，组织切片肾小管坏死部分也显著降低. 同时 PLGA-Oltipraz纳米颗粒使用后, 对主要脏器没有造成 
A

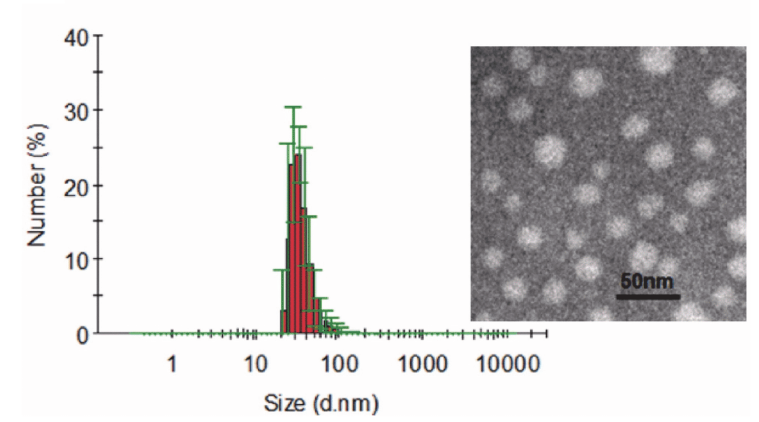

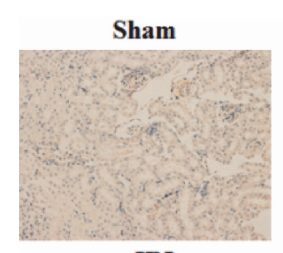

IRI

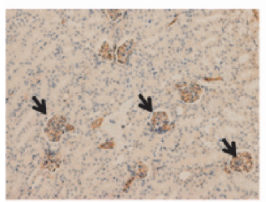

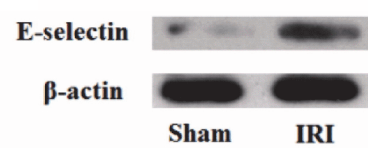

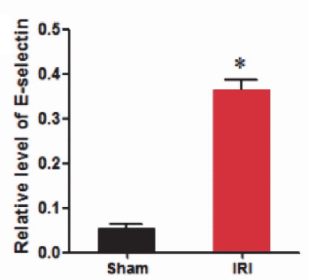

B
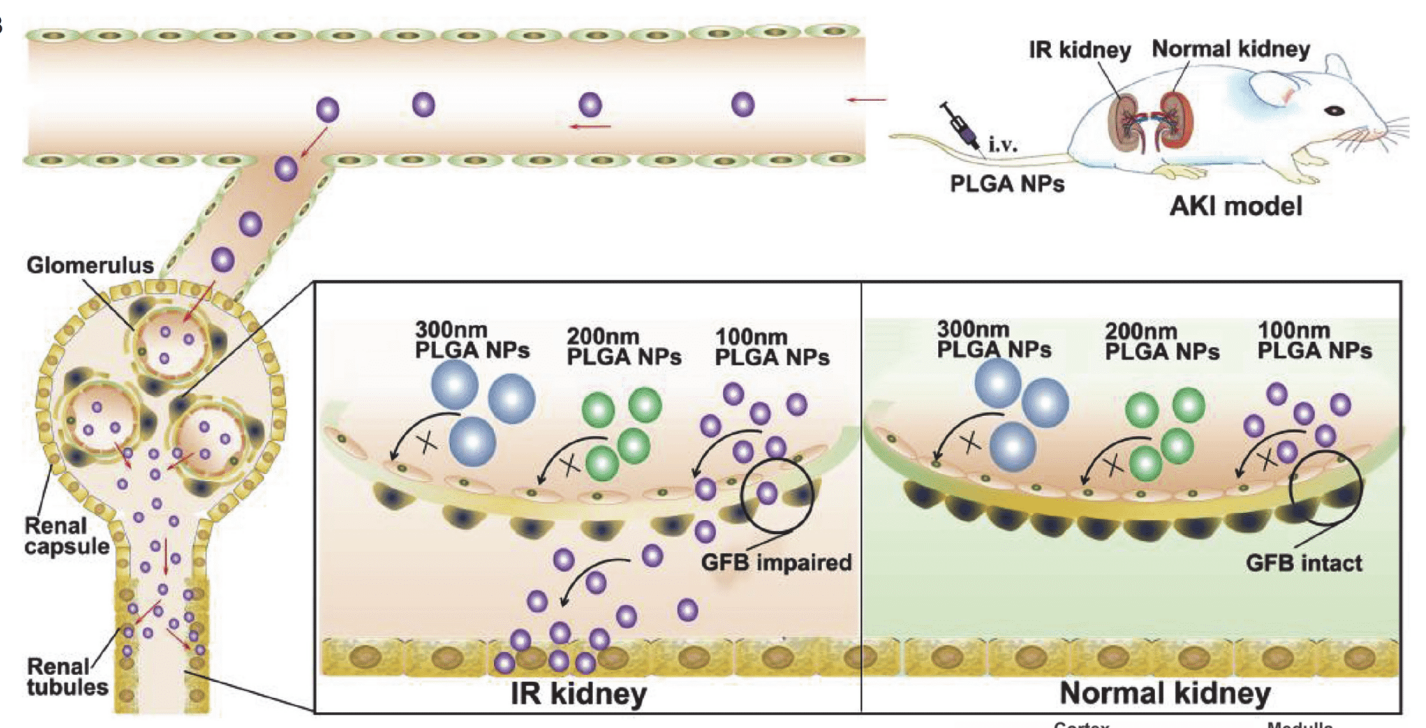

C
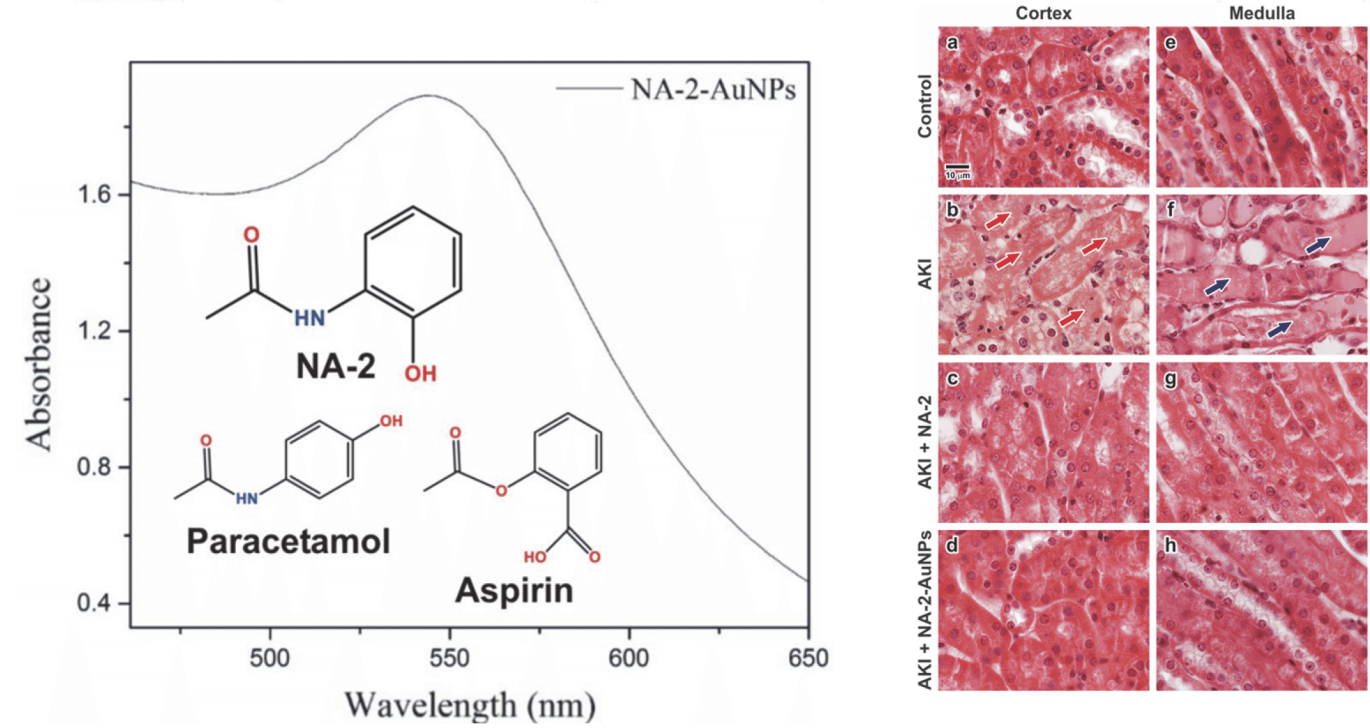

图 2 临床药物用于AKI的治疗. A: 地塞米松用于AKI治疗的示意图 ${ }^{[15]}$; B: 奥替普拉纳米颗粒在AKI中的应用 ${ }^{[16]}$ (已获得Elsevier版权许可); C: N-(2-差基苯基)乙酰胺在AKI治疗中的研究(引自文献[18], 已获得Springer Nature版权许可)(网络版彩图)

Figure 2 Clinical drugs for AKI treatment. A: A schematic diagram of Dexamethasone for AKI treatment ${ }^{[15]}$; B: applications of Oltipraz nanoparticles in $\mathrm{AKI}^{[16]}$ (with permission from Elsevier); C: applications of N-(2-hydroxyphenyl) acetamide nanoparticles in AKI (reproduced from Ref. [18], with permission from Springer Nature) (color online) 
明显的损伤, 为AKI和肾纤维化的治疗提供了新方法.

$\mathrm{N}$-(2-差基苯基)乙酰胺(N-(2-hydroxyphenyl)acetamide, NA-2), 是水杨酸的衍生物. 已有研究表明, NA-2 具有强大的抗炎作用 ${ }^{[17]}$. Siddiqui等人 ${ }^{[18]}$ 将NA-2 偶联 在金纳米颗粒上制备了NA-2-AuNPs(图2C). H\&E(hematoxylin-eosin)切片显示, NA-2-AuNPs预处理对肾脏 损伤具有良好的保护作用. 同样与AKI组相比, NA-2和 NA-2-AuNPs处理显著降低了血清尿素、肌酐的水平 和诱导型一氧化氮合酶(inducible nitric oxide synthase, iNOS)、NF-kB基因的表达. 通过偶联在金纳米颗粒上 的策略将NA-2更好地递送到特定位点，提高生物利用 度同时降低了细胞毒性.

\section{2 天然产物}

自然界中许多天然产物具有强的抗氧化能力, 能 够作为抗氧化剂应用于AKI治疗中，但这些天然产物 的疏水结构限制了其在生物医学中的应用，需要通过 制备成纳米颗粒改变其疏水性质，进而作为一种有效 的抗氧化剂用于 $A K I$ 的治疗. N-甲基-D-天冬氨酸 $(\mathrm{N}$ methyl-D-aspartic acid，NMDA)是一种兴奋性神经递 质. 之前的研究表明, 肾脏内NMDA受体过度活跃可 使一系列相关蛋白去磷酸化，进而发生DAPK途径介 导的细胞死亡 ${ }^{[19]}$. 白藜芦醇(resveratrol, Res) 是一种具 有抗氧化性能的天然产物, Res能够通过ROS清除改善 急性肾损伤. Xu等人 ${ }^{[20]}$ 探讨了 Res和NMDA受体抑制 剂(DAP5)共装载在纳米颗粒中对肾脏缺血再灌注(ischemic reperfusion，I/R)损伤的治疗作用及其机制(图 3A). 研究表明, Res-DAP5-NP能够降低血清生物标志 物的水平. 同时, Res-DAP5-NP通过促进p-DAPK的表 达，抑制p-CaMK和p-AKT的表达来抑制细胞调亡. 体 内治疗实验表明，急性肾损伤显著增加了相关炎症因 子肿瘤坏死因子- $\alpha$ 、白细胞介素- 6 、白细胞介素- $1 \beta$ 等 的表达，而Res-DAP5-NP处理组显著降低了这些炎症 因子的表达. 同样, H\&E肾脏组织切片表明, 在AKI损 伤后，肾脏组织出现严重的肾小管扩张、细胞质间隙 和细胞坏死等. 然而, 与AKI处理组相比, Res-DAP5NP组的细胞形态损伤较小. 这些结果表明, Res-DAP5NP处理可显著降低AKI引起的肾脏损伤.

黑色素是一种存在于多种生物体内的生物聚合 物，具有良好的生物相容性、强的近红外吸收和特殊 的螯合金属离子能力，在癌症的光热治疗和分子成像
中有广泛的应用. 但更值得注意的是，黑色素中含有 非常丰富的抗氧化基团，因此其在AKI抗氧化治疗中 有非常良好的前景. 最近, Cai团队 ${ }^{[21]}$ 使用一种简单的 方法制备了超小尺寸的锰离子螯合黑色素纳米颗粒 $\left(\mathrm{Mn}^{2+}\right.$-chelated melanin with polyethylene glycol, MMPP)(图3B). MMPP在AKI模型小鼠中表现出良好 的生物稳定性和ROS清除能力. T1磁共振 (magnetic resonance, MR)和正电子发射断层成像(positron emission tomography, PET)显示, 经尾静脉注射后, 肾脏对超小 尺寸的纳米颗粒具有优异的摄取能力. 通过尾静脉注 射MMPP纳米颗粒进行 AKI治疗, MMPP纳米颗粒处 理组的尿素氮和血清肌酐水平显著降低, 高注射剂量 下恢复了正常水平. 此外, H\&E肾组织切片显示, AKI 组严重损伤, MMPP纳米颗粒处理下肾脏受损的结构 明显减少. 通过双氢乙二胺(dihydroethidium, DHE)组 织染色共聚焦成像评价肾脏超氧化物的水平, MMPP 纳米颗粒组显示出明显的ROS抑制作用，证实了 MMPP纳米颗粒在AKI治疗中具有较强的抗氧化防御 能力, 从而通过抗氧化作用来缓解AKI. 同时在高剂量 注射MMPP后, H\&E组织切片显示, 肾脏和其他主要器 官没有明显的损伤, 证实了其良好的安全性. MMPP作 为一种多功能的纳米系统，首次用于PET/MR双模成 像引导AKI治疗，也可用于其他氧化应激相关损伤疾 病的治疗.

姜黄素(curcumin)是一种具有广泛的生物功能的 疏水天然化合物. 姜黄素有非常强的抗氧化活性, 从而 能有效保护肾小管上皮细胞免受 $\mathrm{H}_{2} \mathrm{O}_{2}$ 诱导的氧化应 激损伤，但疏水性导致其在体内生物利用率差而限制 了其临床应用. Chen等人 ${ }^{[22]}$ 利用双硬脂酰磷脂酰乙 醇-聚乙二醇(distearoylphosphatidylethanolamine-polyethylene glycol, DSPE-PEG)作为纳米载体装载天然抗 氧化剂姜黄素合成了姜黄素-纳米颗粒, 克服了姜黄素 水溶性差、体内生物利用度低等缺点. 研究结果显示, 相较于游离姜黄素, 姜黄素-NP处理组增强了AKI治疗 效果, 更大程度上降低了血清肌酸激酶(creatine phosphokinase, CPK)、肌䣶(creatinine, $\mathrm{Cr}$ )、尿素和肾小管 的组织学损伤, 表明负载姜黄素的纳米颗粒在体内可 提高由横纹肌溶解诱导的AKI的治疗效果.

\section{3 生物分子}

生物分子通常具有良好的生物相容性和生物可降 

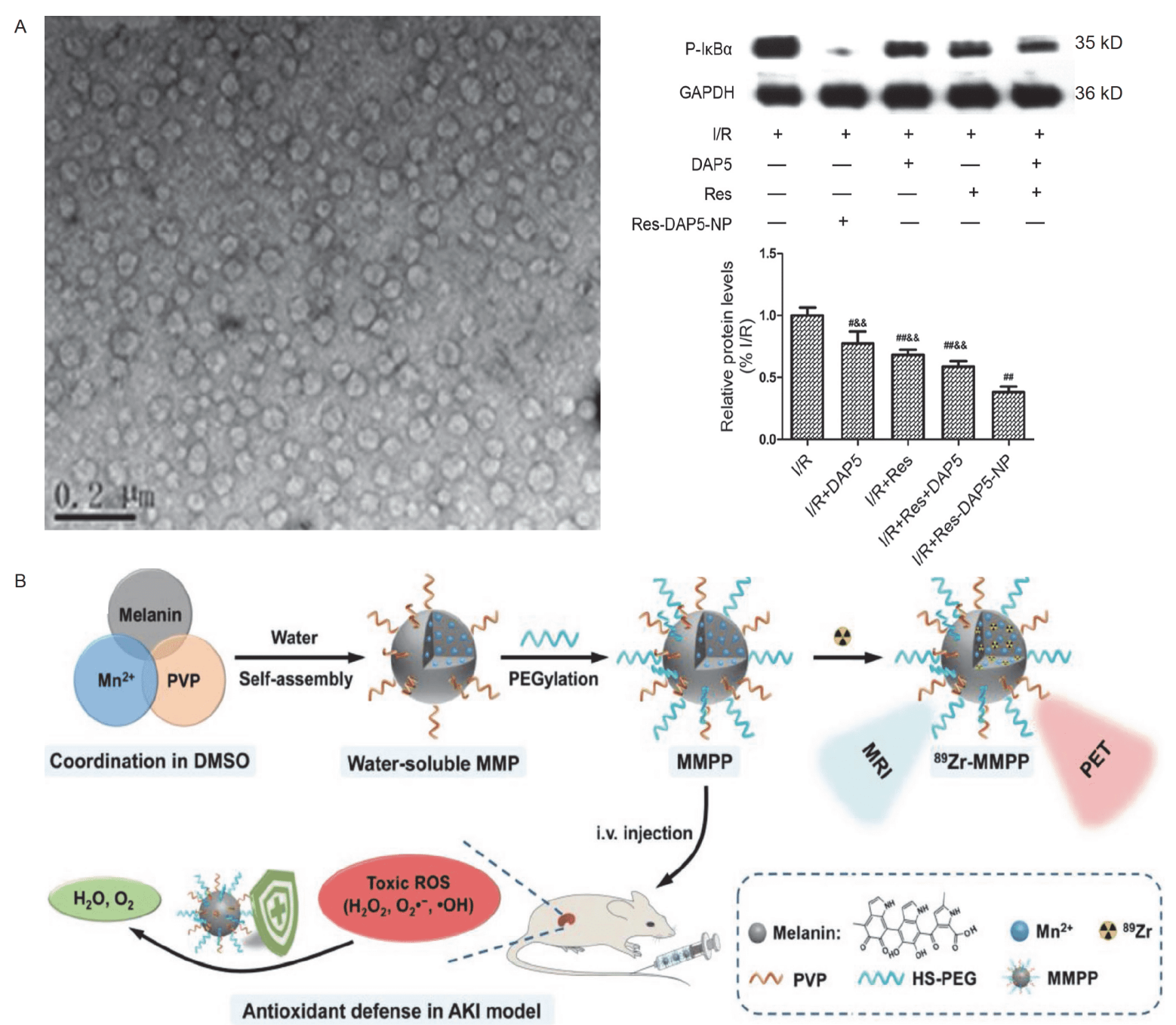

图 3 天然产物用于AKI的治疗. A: 白藜芦醇和NMDA受体抑制剂在AKI疾病中的作用机制 ${ }^{[20]}$; B: 锰离子螯合黑色素纳米颗 粒的合成和AKI治疗(引自文献[21], 已获得Wiley版权许可)(网络版彩图)

Figure 3 Natural products for AKI treatment. A: The mechanism of action of resveratrol and NMDA receptor inhibitors in AKI ${ }^{[20]}$; B: schematic of the synthesis of $\mathrm{Mn}^{2+}$-chelated melanin with polyethylene glycol nanoparticles and AKI therapy (reproduced from Ref. [21], with permission from Wiley) (color online)

解性, 避免了其在体内富集造成的长期毒性, 因此在生 物医学领域具有非常广阔的应用前景. DNA折纸技术 利用DNA分子内和分子间的互补配对，可以设计出不 同大小及形状的结构, 不仅结构灵活多变, 而且单体可 以通过载入噬菌体进行大规模的生产 ${ }^{[23,24]}$. DNA折纸 纳米结构(DNA origami nanostructures, DONs)在生物 体内有非常良好的性质，如毒性小、免疫原性低以及 稳定性良好, 使其成为一种优异的药物载体. 2018年, Cai团队 ${ }^{[25]}$ 首次探索了三种形状的DNA折纸纳米结构 DONs 在体内的生物分布, 并通过PET成像发现, 正常 小鼠和AKI小鼠的肾脏中都会富集大量的DONs(图
4A). 同时肾脏定量PET成像、血清检测、肾H\&E组织 切片证实, 矩形结构的DON(Rec-DON)对AKI小鼠有 明显的治疗效果. 与传统使用 $\mathrm{N}$-乙酰半胱氨酸药物的 治疗相比, Rec-DON可通过DNA碱基的氧化作用, 有 效中和多种活性氧, 进一步保护细胞不受氧化应激的 影响，同时在肾脏中有更多的积累，因此有更理想的 治疗效果.

SS-31是一种线粒体靶向肽, 能够选择性地与仅在 线粒体内膜上表达的心磷脂结合，从而保护线粒体嵴 结构, 促进氧化磷酸化 ${ }^{[26]}$. SS-31具有很强的抗氧化活 性，但其较差的生物分布和递送效率也限制了其在 
A Intravenous injection of Preferential accumulation DONs
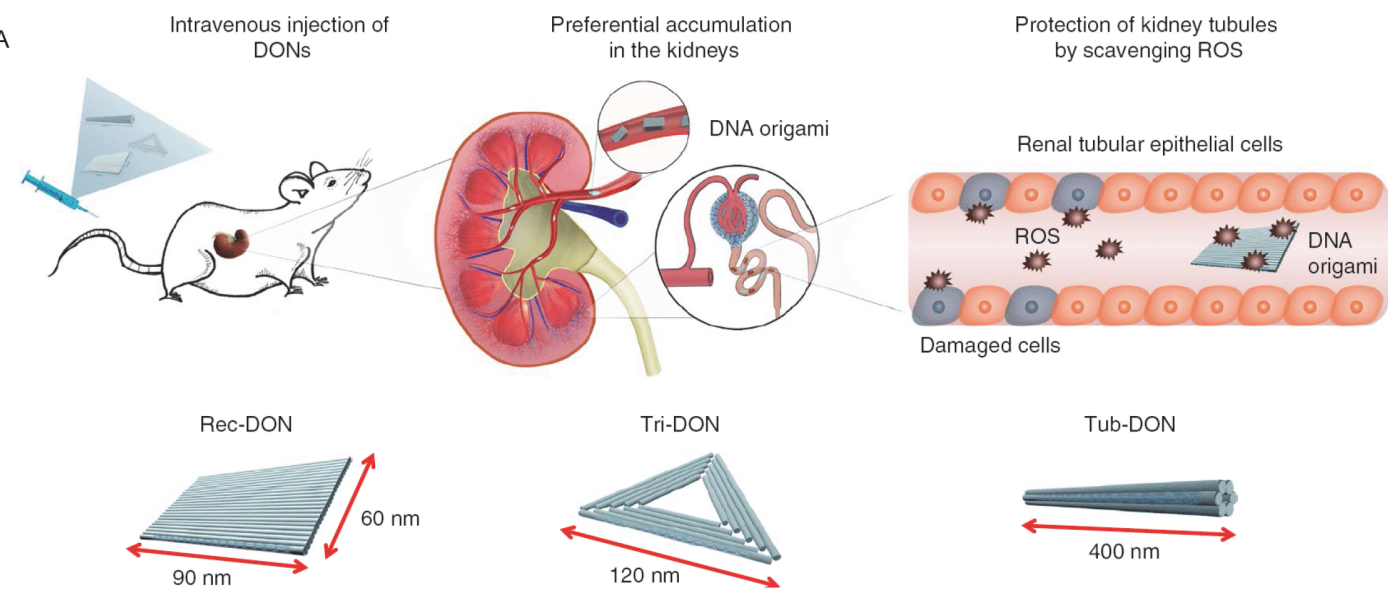

Tub-DON

B
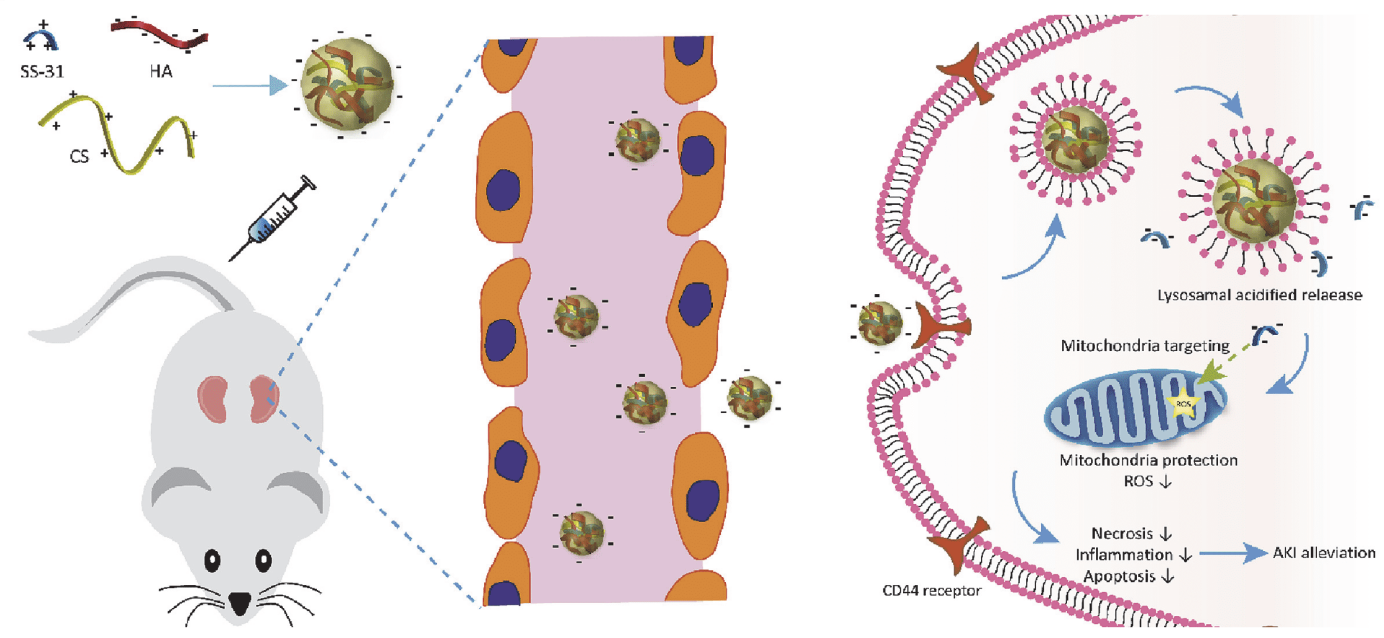

C

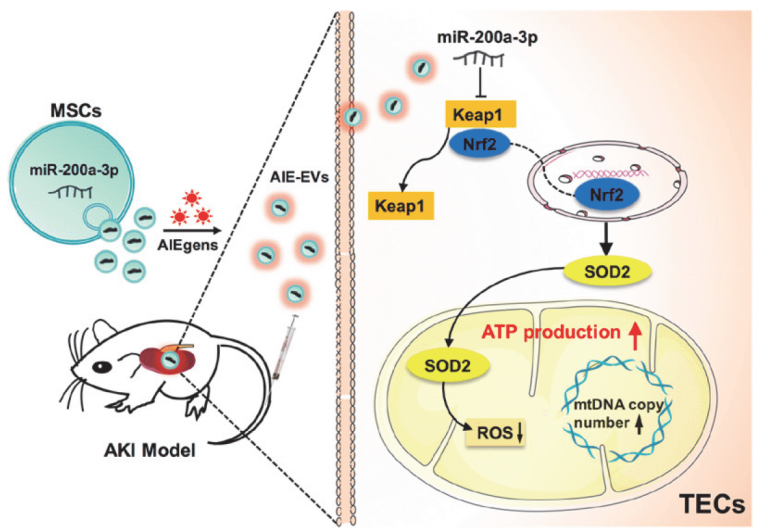

$\mathrm{D}$

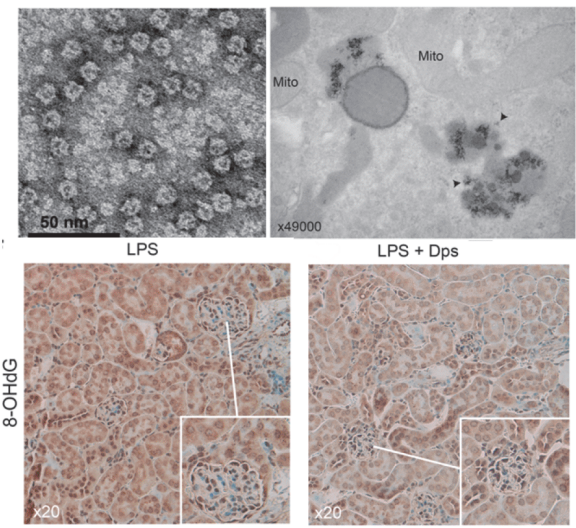

图 4 生物分子用于AKI的治疗. A: 三种不同DNA折纸纳米结构应用于小鼠急性肾损伤模型(引自文献[25], 已获得Springer Nature版权许可); B: 利用静电相互作用设计了由SS-31多肽、阴离子透明质酸和阳离子壳聚糖组成的纳米多聚物(引自文献 [27], 已获得Elsevier版权许可); C: 间充质干细胞来源的囊泡在肾脏修复中的无创、精确的体内可视化(引自文献[29], 已获得 American Chemical Society版权许可); D: DNA结合蛋白在AKI中的应用 ${ }^{[30]}$ (网络版彩图)

Figure 4 Biomolecules for AKI treatment. A: Three different DONs applied to murine models of AKI (reproduced from Ref. [25], with permission from Springer Nature). B: nanopolyplexes consisted of SS-31, anionic HA and cationic chitosan are designed by utilizing electrostatic interaction (reproduced from Ref. [27], with permission from Elsevier); C: noninvasive and precise in vivo visualization of MSC-EVs in kidney repair (reproduced from Ref. [29], with permission from American Chemical Society); D: applications of Dps in AKI ${ }^{[30]}$ (color online) 
AKI治疗中的使用. 2019年，Liu等人 ${ }^{[27]}$ 使用阴离子透 明质酸(hyaluronic acid, HA)和阳离子壳聚糖进行静电 结合用来有效递送SS-31(图4B). 在AKI中, 受损肾脏细 胞的细胞膜表面会过量表达CD44受体, 静脉注射负载 SS-31的聚合物纳米颗粒经过血液循环到达肾脏部位 后, HA 与CD44受体结合, 纳米颗粒通过内吞作用进入 受损细胞，在溶酶体的低 $\mathrm{pH}$ 下，NPs的静电平衡被打 破，SS-31释放并随后靶向线粒体. 苂光成像显示，在 体外与游离SS-31相比, NPs处理组活性氧水平更低, 线粒体结构更加完整. 流式细胞术结果显示, 在体外, NPs很大程度上减少了AKI引起的细胞调亡. 体内实验 表明，NPs处理组显著降低了血清肌酐和尿素氮的水 平, 组织病理学图像显示, NPs处理组肾脏损伤明显修 复，很大程度缓解了AKI造成的严重的肾小管损伤和 坏死. 同时利用透射电子显微镜(transmission electron microscope, TEM)观察了近端肾小管细胞线粒体结构. TEM图像显示, 生理盐水处理组有严重的线粒体损伤, 线粒体内外膜破裂，嵴缺失. 与此相反，经NPs处理的 动物线粒体膜内结构完整, 崉结构规整, 几乎没有肿胀 的线粒体. 因此, NPs很大程度上能够保护线粒体结构, 减少肾小管细胞凋亡和坏死.

细胞外囊泡(extracellular vesicles, EVs) 分为外 泌体、更小的囊泡和微囊泡, 它们能够参与细胞间 的通讯. 在AKI发生过程中, EVs可以调节免疫应答 和血栓形成 ${ }^{[28]}$. Wang团队 ${ }^{[29]}$ 将EVs 和聚集诱导发光 的成像分子(DPA-SCP) 用于肾脏 I/R损伤模型(图4C). EVs激活了Keap1-Nrf2信号通路发挥抗氧化作用，通 过调节线粒体结构和功能帮助恢复肾脏功能. DPA$\mathrm{SCP}$ 不会影响EVs的再生能力和治疗效果. 因此，在 AKI模型中, 实现了对EVs在体内的无创、精确、安 全的检测.

肾小球滤过屏障的存在给纳米颗粒输送到近端小 管带来了挑战. 绝大部分蛋白由于尺寸过大而不能通 过肾小球滤过屏障. Uchida等人 ${ }^{[30]}$ 报道了来自饥饿细 胞的DNA结合蛋白(Dps)(图4D), 一种非常小的蛋白质 纳米颗粒，该蛋白具有天然的细胞保护特性并且能够 通过肾小球滤过屏障到达肾脏. 通过在Dps上添加锰 团簇(manganese-bound Dps, MnDps)，进一步增强了 其抗氧化能力. $\mathrm{MnDps}$ 可以作为一种强大的抗氧化剂, 在脓毒症诱导的肾脏损伤模型中减轻近端肾小管损 伤, 恢复肾脏功能.

\section{4 无机纳米材料}

相比于天然产物等有机纳米颗粒, 无机纳米材料 具有独特的光学、电学和磁学等性质, 在多种疾病的 检测、成像和治疗中都有非常广泛的引用 ${ }^{[31,32]}$. 许多 具有抗氧化作用的无机纳米颗粒广泛用于脑缺血性损 伤的治疗, 同样也可以用于AKI疾病的治疗. 钼基多金 属氧酸盐(polyoxometalate, POM) 是一种拥有两种价态 $\left(\mathrm{Mo}^{5+}\right.$ 和 $\left.\mathrm{Mo}^{6+}\right)$ 的无机纳米材料 ${ }^{[33]}, \mathrm{POM}$ 中 Mo 离子价态 的比例在一定的还原或氧化条件下很容易发生变化, 因此具有清除有害活性氧的能力. Cai团队 ${ }^{[34]}$ 使用一种 快速、低成本和大规模的方法合成超小的POM作为 一种新型的抗氧化剂(图5A), POM的粒径均一且小于 $10 \mathrm{~nm}$, 因此能够通过肾小管滤过膜累积在肾脏组织. $\mathrm{POM}$ 高度亲水, 能够在生理环境下稳定存在。体外实 验结果表明，POM对肾脏细胞没有明显毒性，同时相 比于对照组, POM处理组具有更低的活性氧水平. 通 过强PET成像评估POM纳米团簇在AKI小鼠体内的生 物分布, 发现其能够在肾脏有良好的富集并且在较长 一段时间显示出高累积量. POM在血液循环过程中能 够通过通过肾小球并且在一定时间后通过肾脏排出体 外. 动态PET成像结果显示, AKI小鼠由于具有严重的 肾脏损伤, 因此 ${ }^{68} \mathrm{Ga}$-EDTA滞留在肾脏, 而POM处理组 的动态曲线分布恢复至正常情况. 同时经POM治疗后, 小鼠体重、血尿素氮(blood urea nitrogen, BUN)、血 清肌酲 $(\mathrm{Cr})$ 、肾损伤分子-1(kidney injury molecule-1, KIM-1)和血红素氧合酶-1(heme oxygenase-1, HO-1)恢 复至正常水平, H\&E肾组织切片同样显示, AKI小鼠肾 脏的损伤得到了明显的抑制. 此外，POM处理后可显 著减少肾脏中 8-羟基-2-脱氧鸟苷(8-hydroxy-2-deoxyguanosine, 8-OHdG)的含量, 说明POM纳米团簇还能抑 制DNA损伤和脂质过氧化. 因此, 这种超小POM纳米 团簇对AKI或其他由活性氧引发的疾病有非常广阔的 应用前景.

黑磷(black phosphorus, BP)作为一种二维材料以 其优异的光学性质在生物医学领域表现出了巨大的潜 力, 特别是在癌症的光热治疗、光动力治疗和一些神 经退行性疾病等已经有了非常广泛的应用 ${ }^{[35,36]}$. 最近, Wang团队 ${ }^{[37]}$ 的研究表明, 黑磷纳米片(BP nanosheets, BPNSs)由于特殊的几何结构, 在肾脏有良好的富集. 同时黑磷具有足够的还原分子，在水、氧和光照的共 


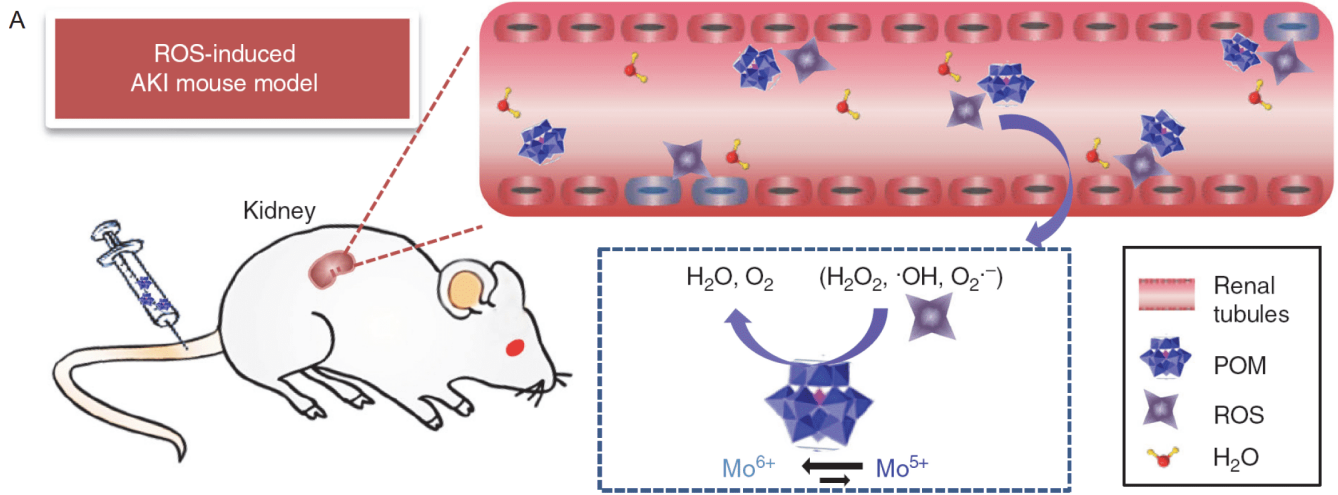

B

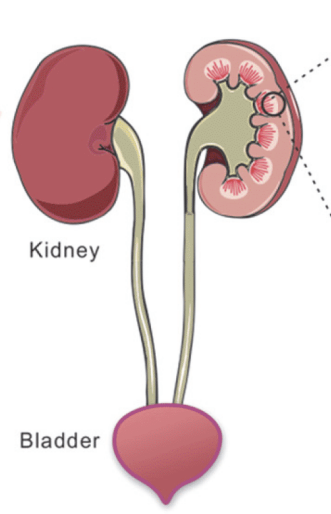

Intravenous injection of Atv/PTP-TCeria
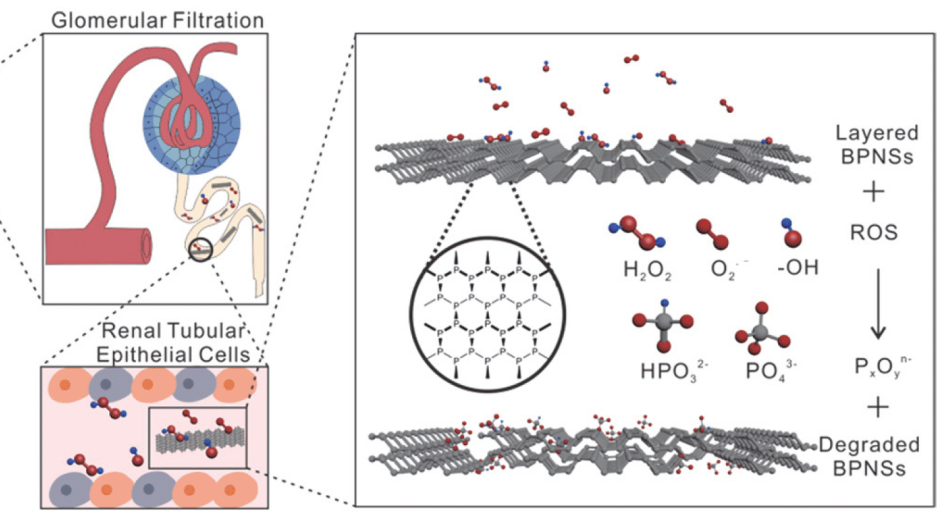

C

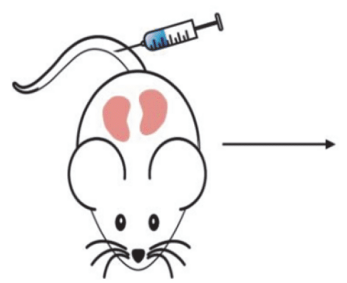
Preferential accumulation in the kidneys

\section{Protection of kidney tubules by scavenging ROS}

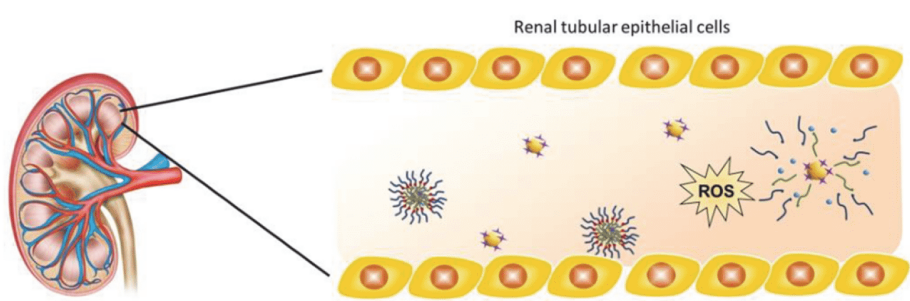

图 5 无机纳米颗粒用于AKI的治疗. A: AKI中钼基多金属氧酸盐的应用 ${ }^{[34]}$; B: 黑磷在肾脏积累和缓解AKI的示意图(引自文 献[37], 已获得American Chemical Society版权许可); C: 二氧化铈纳米颗粒负载阿托伐他(Atv/PTP-TCeria NPs)靶向肾脏线粒 体, 释放药物清除过量ROS ${ }^{[38]}$ (网络版彩图)

Figure 5 Inorganic nanoparticles for AKI treatment. A: The applications of POM nanoclusters in AKI ${ }^{[34]}$; B: a schematic illustration of BP accumulation in kidney and its function in alleviating AKI (reproduced from Ref. [37], with permission from American Chemical Society); C: Atv/ PTP-TCeria NPs could target the mitochondria of kidney and release drugs to scavenge excessive ROS $^{[38]}$ (color online)

同作用下，很容易被氧化成磷氧化物(图5B). 通过这种 作用, 黑磷纳米片既可以作为肾脏靶向的分子, 也能作 为抗氧化剂用于清除肾脏中过量的ROS．体外实验表 明，BPNSs对于肾脏细胞有着较低毒性，同时苂光共 聚焦成像和流式结果表明，BPNSs能被肾脏细胞吞噬 从而保护肾脏细胞免受ROS损害。注射后 $12 \mathrm{~h}$ 收集 AKI小鼠的肾脏和其他器官，通过苂光信号量化各器 官的摄取情况，收集到的心、肝、脾、肺等cy5的信号 相对较低, 而肾脏BPNSs的信号相对较高, 表明BPNSs 具有良好的肾脏靶向性. 同时血清生物标志物检测和 $H \& E$ 肾组织切片结果显示, BPNSs处理后AKI小鼠的 各项指标均有恢复. BPNSs用于肾脏治疗的优点在于 其具有非常高的ROS清除能力和较低的体内细胞毒 性, 并且绝大部分的纳米片可以从体内代谢出去, 从而 
实现高效低毒AKI治疗.

氧化铈纳米颗粒具有极强的ROS清除活性，因此 在治疗活性氧相关疾病方面已有广泛的研究. 二氧化 铈纳米颗粒不能靶向线粒体，为了提高治疗效率， $\mathrm{Du}$ 团队 ${ }^{[38]}$ 设计了一种二氧化铈纳米颗粒负载阿托伐他 (atorvastatin, Atv)的ROS响应的纳米给药系统用于AKI 的治疗 (图 5C). 三苯基膦修饰二氧化铈纳米颗粒(TCeria NPs)后在氧化铈表面涂覆ROS响应的有机聚合物 (mPEG-TK-PLGA)和阿托伐他汀(Atv/PTP-TCeria NPs). 研究显示, Atv/PTP-TCeria NPs可在肾脏内积累, 在较强的ROS响应下释放Atv, 从而靶向线粒体清除过 量ROS. Atv/PTP-TCeria NPs能有效降低AKI小鼠的氧 化应激和炎症反应, 保护线粒体结构, 减少细胞凋亡和 坏死. 这种ROS响应的纳米给药系统在脓毒症诱导的 AKI治疗中具有良好的潜力.

\section{2 结论与展望}

本文系统总结了近年来具有ROS清除功能的纳 米材料在AKI治疗中的应用，包括药物递送、天然产 物、生物分子和无机纳米材料. 这一新型治疗策略和
这些新型纳米材料将在AKI的治疗中发挥重要作用. 虽然抗氧化治疗策略为AKI的治疗提供了新途径, 也 已经取得了一定的进展，但仍然存在一些问题和 挑战.

发展新的抗氧化纳米材料. 虽然目前存在非常多 的纳米材料可用于AKI的抗氧化治疗, 但其通常存在 材料制备工艺复杂、成本高难以大面积推广的问题. 另外, 一旦注射进入血液循环后, 它们会随血液循环进 入全身器官, 可能被单核吞噬细胞吞噬, 或者在进入血 液时与蛋白质结合, 纳米颗粒与蛋白质之间的相互作 用会改变蛋白质分子的构象，从而影响血液蛋白质的 生物学功能. 因此, 迫切需要开发出能够大量制备、 生物安全性好, 具有良好靶向作用和强ROS清除能力 的纳米材料用于 AKI的治疗. 此外, 纳米材料进入血液 循环后的行为仍然需要深入探究.

发展新的联合AKI治疗策略. 虽然AKI的抗氧化策 略取得了一定的进展, 但仅靠这一特异性的治疗太过 单一, 治疗效果有限. 需要基于AKI基础的病理机制, 发展更多特异性的疗法联合抗氧化机制用于AKI治疗, 例如实现快速便捷地清除血液中多余的含氮代谢废 物, 以及AKI模型中肾脏免疫微环境的调控等.

\section{参考文献}

1 Bellomo R, Kellum J A, Ronco C. Acute kidney injury. Lancet, 2012, 380: 756-766

2 Ricci Z, Cruz D, Ronco C. The RIFLE criteria and mortality in acute kidney injury: a systematic review. Kidney Int, 2008, 73: 538-546

3 Prowle J R, Chua H R, Bagshaw S M, et al. Clinical review: Volume of fluid resuscitation and the incidence of acute kidney injury: a systematic review. Crit Care, 2012, 16: 230

4 Leung K C W, Tonelli M, James M T. Chronic kidney disease following acute kidney injury—risk and outcomes. Nat Rev Nephrol, 2013, 9: 7785

5 Wang W, Jittikanont S, Falk S A, et al. Interaction among nitric oxide, reactive oxygen species, and antioxidants during endotoxemia-related acute renal failure. Am J Physiol Renal Physiol, 2003, 284: F532-F537

6 Hsu C Y, McCulloch C E, Fan D, et al. Community-based incidence of acute renal failure. Kidney Int, 2007, 72: 208-212

7 Gonsalez S R, Cortês A L, Silva R C, et al. Acute kidney injury overview: from basic findings to new prevention and therapy strategies. Pharmacol Ther, 2019, 200: 1-12

8 Sun J, Zhang J, Tian J, et al. Mitochondria in sepsis-induced AKI. J Am Soc Nephrol, 2019, 30: 1151-1161

9 Tran S, DeGiovanni P J, Piel B, et al. Cancer nanomedicine: a review of recent success in drug delivery. Clin Transl Med, 2017, 6: 44

10 Cheng L, Wang C, Feng L, et al. Functional nanomaterials for phototherapies of cancer. Chem Rev, 2014, 114: 10869-10939

11 Sharfuddin A A, Molitoris B A. Pathophysiology of ischemic acute kidney injury. Nat Rev Nephrol, 2011, 7: 189-200

12 Coca S G, Singanamala S, Parikh C R. Chronic kidney disease after acute kidney injury: a systematic review and meta-analysis. Kidney Int, 2012, 81: $442-448$

13 Moreau P, Masszi T, Grzasko N, et al. Oral ixazomib, lenalidomide, and dexamethasone for multiple myeloma. N Engl J Med, 2016, 374: 16211634 
14 Durie B G M, Hoering A, Abidi M H, et al. Bortezomib with lenalidomide and dexamethasone versus lenalidomide and dexamethasone alone in patients with newly diagnosed myeloma without intent for immediate autologous stem-cell transplant (SWOG S0777): a randomised, open-label, phase 3 trial. Lancet, 2017, 389: 519-527

$15 \mathrm{Hu}$ J B, Song G L, Liu D, et al. Sialic acid-modified solid lipid nanoparticles as vascular endothelium-targeting carriers for ischemia-reperfusioninduced acute renal injury. Drug Deliver, 2017, 24: 1856-1867

16 Yu H, Lin T, Chen W, et al. Size and temporal-dependent efficacy of oltipraz-loaded PLGA nanoparticles for treatment of acute kidney injury and fibrosis. Biomaterials, 2019, 219: 119368

17 Perveen K, Hanif F, Jawed H, et al. Protective efficacy of N-(2-Hydroxyphenyl) acetamide against adjuvant-induced arthritis in rats. Biomed Res Int, 2013, 2013: 1-8

18 Siddiqui R A, Simjee S U, Kabir N, et al. N-(2-hydroxyphenyl)acetamide and its gold nanoparticle conjugation prevent glycerol-induced acute kidney injury by attenuating inflammation and oxidative injury in mice. Mol Cell Biochem, 2019, 450: 43-52

19 Palomeque J, Rueda O V, Sapia L, et al. Angiotensin II-induced oxidative stress resets the $\mathrm{Ca}^{2+}$ dependence of $\mathrm{Ca}^{2+}$-calmodulin protein kinase II and promotes a death pathway conserved across different species. Circ Res, 2009, 105: 1204-1212

$20 \mathrm{Xu} \mathrm{Y,} \mathrm{Zhang} \mathrm{B,} \mathrm{Xie} \mathrm{D,} \mathrm{et} \mathrm{al.} \mathrm{Nanoparticle-mediated} \mathrm{dual} \mathrm{delivery} \mathrm{of} \mathrm{resveratrol} \mathrm{and} \mathrm{DAP5} \mathrm{ameliorates} \mathrm{kidney} \mathrm{ischemia/reperfusion} \mathrm{injury} \mathrm{by}$ inhibiting cell apoptosis and inflammation. Oncotarget, 2017, 8: 39547-39558

21 Sun T, Jiang D, Rosenkrans Z T, et al. A melanin-based natural antioxidant defense nanosystem for theranostic application in acute kidney injury. Adv Funct Mater, 2019, 29: 1904833

22 Chen X, Sun J, Li H, et al. Curcumin-loaded nanoparticles protect against rhabdomyolysis-induced acute kidney injury. Cell Physiol Biochem, 2017, 43: 2143-2154

23 Zhang F, Jiang S, Wu S, et al. Complex wireframe DNA origami nanostructures with multi-arm junction vertices. Nat Nanotech, 2015, 10: 779784

24 Liu X, Zhang F, Jing X, et al. Complex silica composite nanomaterials templated with DNA origami. Nature, 2018, 559: 593-598

25 Jiang D, Ge Z, Im H J, et al. DNA origami nanostructures can exhibit preferential renal uptake and alleviate acute kidney injury. Nat Biomed Eng, 2018, 2: 865-877

26 Sweetwyne M T, Pippin J W, Eng D G, et al. The mitochondrial-targeted peptide, SS-31, improves glomerular architecture in mice of advanced age. Kidney Int, 2017, 91: 1126-1145

27 Liu D, Jin F, Shu G, et al. Enhanced efficiency of mitochondria-targeted peptide SS-31 for acute kidney injury by pH-responsive and AKI-kidney targeted nanopolyplexes. Biomaterials, 2019, 211: 57-67

28 Karpman D, Loos S, Tati R, et al. Haemolytic uraemic syndrome. J Intern Med, 2017, 281: 123-148

29 Cao H, Cheng Y, Gao H, et al. In vivo tracking of mesenchymal stem cell-derived extracellular vesicles improving mitochondrial function in renal ischemia-reperfusion injury. ACS Nano, 2020, 14: 4014-4026

30 Uchida M, Maier B, Waghwani H K, et al. The archaeal Dps nanocage targets kidney proximal tubules via glomerular filtration. J Clin Invest, 2019, 129: 3941-3951

31 Mu J, Lin J, Huang P, et al. Development of endogenous enzyme-responsive nanomaterials for theranostics. Chem Soc Rev, 2018, 47: 5554-5573

32 Lim E K, Kim T, Paik S, et al. Nanomaterials for theranostics: recent advances and future challenges. Chem Rev, 2015, 115: 327-394

33 Yong Y, Zhou L, Zhang S, et al. Gadolinium polytungstate nanoclusters: a new theranostic with ultrasmall size and versatile properties for dualmodal MR/CT imaging and photothermal therapy/radiotherapy of cancer. NPG Asia Mater, 2016, 8: e273

34 Ni D, Jiang D, Kutyreff C J, et al. Molybdenum-based nanoclusters act as antioxidants and ameliorate acute kidney injury in mice. Nat Commun, 2018, 9: 5421

35 Shao J, Xie H, Huang H, et al. Biodegradable black phosphorus-based nanospheres for in vivo photothermal cancer therapy. Nat Commun, 2016, 7: 12967

36 Liu H, Du Y, Deng Y, et al. Semiconducting black phosphorus: synthesis, transport properties and electronic applications. Chem Soc Rev, 2015, 44: $2732-2743$

37 Hou J, Wang H, Ge Z, et al. Treating acute kidney injury with antioxidative black phosphorus nanosheets. Nano Lett, 2020, 20: 1447-1454

$38 \mathrm{Yu} \mathrm{H}$, Jin F, Liu D, et al. ROS-responsive nano-drug delivery system combining mitochondria-targeting ceria nanoparticles with atorvastatin for acute kidney injury. Theranostics, 2020, 10: 2342-2357 


\title{
Nanomaterials with ROS scavenging ability to alleviate acute kidney injury
}

\author{
HOU LinQian, ZHANG Rui, ZHONG XiaoYan \& CHENG Liang \\ Collaborative Innovation Center of Suzhou Nano Science and Technology, Institute of Functional Nano \& Soft Materials (FUNSOM), \\ Soochow University, Suzhou 215123, China
}

Acute kidney injury (AKI) is a critical disease associated with high morbidity and mortality. Apart from hemodialysis and kidney transplantation, there is no targeted therapy. Therefore, it is necessary to start from the pathogenesis of AKI and explore new treatment strategies. Recent studies have shown that oxidative stress is an important pathological mechanism of AKI. Therefore, alleviating renal oxidative stress has become a key issue in the treatment of AKI. The development of functional nanomaterials with reducibility provides new ideas for AKI treatment. Here we reviewed the recent studies in the application of functional nanomaterials with active oxygen scavenging ability in the treatment of AKI.

acute kidney injury, reducing materials, ROS scavenging, nanoparticles

doi: $10.1360 /$ SSV-2020-0237 